\title{
DÉGRADATION DE LA MATIËRE GRASSE PAR LIPOLYSE
}

\author{
par \\ P. JAMOTTE \\ Station laitière de Gembloux (Belgique)
}

On rencontre dans les laits et les crèmes des micro-organismes capables d'hydrolyser la matière grasse.

Cependant la lipolyse, constatée fréquemment dans des produits crus, peu contaminés, ne provient généralement pas de l'activité des germes lipolytiques.

La lipolyse est due, le plus souvent, à l'action d'enzymes présents dans les produits laitiers eux-mêmes. Ce sont les lipases naturelles du lait qui dégradent la matière grasse. Au stade final de leur action, elles décomposent les glycérides en glycérol et acides gras. La dégradation est souvent partielle avec production des mono et digly cérides intermédiaires. Ces derniers sont responsables de l'amertume décelée dans les produits laitiers.

Le défaut " rance " apparaît lorsque des acides gras sont libérés (acide butyrique). Lorsque la dégradation est poussée assez loin, le défaut s'amplifie jusqu'à l'apparition du goût de savon. La lipolyse des crèmes se marque par des modifications de la tension superficielle (d'où allongement des durées de barattage) et un certain freinage de l'acidification lactique.

La lipolyse se développe uniquement dans les produits crus, non chauffés, non pasteurisés. Les lipases naturelles sont en effet détruites par un traitement thermique peu sévère. Leur action est activée au maximum en milieu neutre ou peu acide.

La lipase normale est liée aux protéines du plasma. Pour exercer une action perceptible sur la matière grasse, cette lipase du plasma doit être adsorbée par les membranes des globules gras.

Cette adsorption induit l'action lipolytique. Elle est réalisée par les traitements trop violents du lait ou de la crème : actions mécaniques (agitation incorporation d'air) ou chocs thermiques (réchauffement et refroidissement intempestifs). La libération d'une acidité de matière grasse appréciable reflète les erreurs de techniques commises à la production ou à la préparation des produits crus. Les taux d'acidité constatés correspondent assez fréquemment aux 
intensités des défauts de goût. Il y a cependant des exceptions (cas de lipolyse induite non dommageable).

La relation entre le défaut de rancidité et un taux élevé d'acidité libre est meilleur lorsque les produits sont affectés par une autre lipase naturelle des laits, présente et active au niveau des membranes globulaires.

Cette lipase anormale se retrouve dans les laits de vaches en fin de lactation ou atteintes de troubles physiologiques. Ces laits ou crèmes deviennent spontanément rances et ce, de façon rapide, parfois même explosive. Cette lipase dite de membrane se rencontre heureusement, beaucoup plus rarement.

Elle est également thermolabile.

Contrairement aux lipases naturelles précitées, les lipases d'origine microbienne, beaucoup moins fréquentes et par conséquent moins redoutables, seraient cependant plus résistantes aux traitements thermiques [2].

\section{I. - Méthodes d'analyse}

Le développement de la lipolyse dans un lait ou une crème, s'apprécie le plus aisément par des déterminations de l'acidité de la matière grasse (méthode habituelle par titration). Les difficultés résident le plus souvent dans la façon d'extraire cette matière grasse accompagnée des traces d'acides gras en respectant ces dernières.

Divers procédés ont été utilisés et comparés.

Le barattage (en flacon ou en mixer), suivi de délaitage et lavage, reste le procédé de référence le plus recommandable. II présente l'avantage de donner des résultats identiques à ceux obtenus réellement en fabrication beurrière. Il est malheureusement compliqué et assez lent. Il ne convient que pour l'analyse de quelques échantillons isolés [3].

L'extraction aux solvants est, par contre, simple et rapide. Elle s'adapte bien aux grandes séries. Nous la préconisons pour la détermination de la qualité des crèmes [7].

La séparation à la mixture détergente paraît être la méthode suffisamment rapide, convenant parfaitement au contrôle en laboratoire du développement de la lipolyse dans des séries raisonnables de laits ou de crèmes soumis à des traitements expérimentaux [4], [5], [6].

\section{A. Barattage.}

Pour baratter de petites portions de crème on peut utiliser le mixer. L'agitation intense produite par cet appareil en restreint 
l'emploi aux crèmes aptes à se baratter très facilement (c'est-à-dire présentant des teneurs en gras, températures et acidités Dornic favorables). Après l'obtention des grains, retirer le babeurre, ajouter une quantité d'eau glacée, laisser agir cette eau pendant 3-4 minutes, enlever l'eau et reprendre le beurre.

Lorsque les crèmes ne sont pas en bonnes conditions de barattage, il vaut mieux les baratter, à la main, dans un flacon ou bouteille à fermeture mécanique. Tempérer la crème vers $13-15^{\circ} \mathrm{C}$, remplir le flacon au tiers environ, diluer éventuellement une crème trop riche ou trop visqueuse. Baratter par secouements, éliminer le babeurre, remplacer par de l'eau glacée, laver et évacuer l'eau.

On met alors fondre le beurre obtenu en plaçant le flacon dans un bain-marie à $45-50^{\circ} \mathrm{C}$.

Dès que la matière grasse est séparée, reprendre celle-ci dans un tube à centrifuger. Ecarter les mousses et le sérum en centrifugeant pendant 5 minutes, au moins, dans une centrifugeuse chauffée. Ensuite, filtrer la matière grasse sur filtre sec, à l'étuve à $45-50^{\circ} \mathrm{C}$ (ne pas entraîner de phase aqueuse sur le filtre).

La matière grasse limpide collectée dans un petit erlenmeyer, est alors disponible pour une pesée de 5 ou $10 \mathrm{~g}$ (cf. méthode de détermination de l'acidité de la matière grasse du beurre).

Le barattage n'est cependant applicable que pour des crèmes aptes à se laisser baratter assez rapidement. On observe en effet un accroissement des valeurs d'acidité lorsque le barattage est trop lent (une action mécanique prolongée accroît la lipolyse).

\section{B. Extraction aux solvants (FFA).}

Préparer une série de tubes à essai contenant $10 \mathrm{ml}$ d'alcool éthylique à $96^{\circ}$ et $5 \mathrm{ml} \mathrm{H} \mathrm{H}_{2} \mathrm{SO}_{4} \mathrm{~N} / 10$.

Peser $5 \mathrm{~g}$ de crème, préalablement fluidifiée par réchauffage rapide à $35-40^{\circ} \mathrm{C}$.

Mélanger, immédiatement après chaque pesée, la crème aveo l'alcool et l'acide, pendant 30 secondes à 1 minute.

Ajouter ensuite dans toute la série des tubes, $20 \mathrm{ml}$ du mélange suivant :

- 40 parties d'éther di-éthylique,

- 60 parties d'éther de pétrole plus un indicateur (thymolphtaléine, par exemple).

Boucher les tubes.

Agiter mécaniquement la série de tubes pendant une minute environ (agitateur Turbula, par exemple).

Centrifuger les tubes pendant 3 à 5 minutes à $\pm 1000 \mathrm{~T}$ (centrifuge Gerber). 
Titrer l'acidité de la totalité de la couche éthérée surnageante par apports successifs de doses de $0,5 \mathrm{ml}$ de méthylate de $\mathrm{Na} \pm$ $0,0125 \mathrm{~N}$ (au moyen d'une pompe doseuse de laboratoire).

Les résultats sont exprimés en $\mathrm{ml}$ alcali $\mathrm{N}$ pour $100 \mathrm{~g}$ de matière grasse, d'où obligation d'effectuer une détermination rapide de la teneur en matière grasse des crèmes par Gerber-Koehler.

$N$. B. - On peut évidemment aussi exprimer les résultats pour $100 \mathrm{~g}$ de crème.)

\section{Séparation par mixture détergente (BDI).}

Ce procédé consiste à mélanger, à la crème, une mixture détergente. Après séjour d'une demi-heure maximum au bain-marie, à 85 $90^{\circ} \mathrm{C}$, et quelques agitations, la matière grasse se sépare nettement.

L'opération s'effectue soit dans des tubes à centrifuger spéciaux, rétrécis à la portion supérieure, soit dans des ballons à long col et fond plat.

Le choix du récipient dépend du produit à analyser (crème ou lait) et de la quantité de gras désirée pour la titration.

Le mélange détergent est composé comme suit :

$$
\begin{aligned}
& \text { Sodium métaphosphate }\left(\mathrm{NaPO}_{3}\right)_{\mathrm{x}} \ldots \ldots 25 \mathrm{~g} \\
& \text { Triton X } 100 \ldots \ldots \ldots \ldots \ldots \ldots \ldots \ldots . \ldots 12 \mathrm{ml}
\end{aligned}
$$

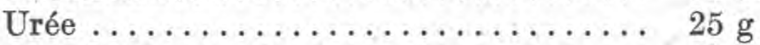

$$
\begin{aligned}
& \text { Alcool isopropylique ........... } 50 \mathrm{ml} \\
& \text { Eau en quantité suffisante pour porter } \\
& \text { au volume de ............... } 500 \mathrm{ml}
\end{aligned}
$$

Le processus d'extraction s'effectue de la façon suivante :

A $20 \mathrm{ml}$ de crème (ou un multiple de $20 \mathrm{ml}$ ), ajouter : $25 \mathrm{ml} \mathrm{du}$ mélange détergent (ou un multiple de $25 \mathrm{ml}$ ).

Bien mélanger intimement les deux produits sans produire trop de mousse. Mettre ensuite les tubes spéciaux (ou les ballons) au bain-marie chaud, réglé à $85-90^{\circ} \mathrm{C}$. Ne jamais dépasser $90^{\circ} \mathrm{C}$, sinon risques de grimpage.

Après une dizaine de minutes de chauffage, mélanger une nouvelle fois prudemment. Remettre au bain-marie. Répéter le mélange, une ou plusieurs fois, selon l'avancement de la séparation.

Dès que la séparation est suffisamment avancée, ajouter de l'eau à $90^{\circ} \mathrm{C}$, environ 10-13 ml (ou un multiple). Mélanger prudemment, sans réémulsionner.

L'addition d'eau chaude assure une séparation nette des deux couches et lave la matière grasse du détergent éventuellement absorbé.

Dès que l'opération est ainsi menée à bonṇe fin (après environ 20-25 minutes) on centrifuge alors les tubes spéciaux pendant 5 minutes maximum. (Cette centrifugation ne s'applique pas aux ballons, voir plus loin.) 
Au sortir de la centrifuge, on rectifie, dans les tubes, le niveau du gras en ajoutant à nouveau un peu d'eau chaude et en centrifugeant à nouveau 2 minutes.

On peut alors prélever la quantité de matière grasse requise pour titration (seringue médicale).

Dans le cas d'emploi de ballons, on procède comme suit : dès que la séparation est suffisante et après avoir ajouté un complément d'eau chaude, on prélève à la seringue la couche supérieure contenant le gras et un peu de la couche trouble sous-jacente. Ce prélèvement est mis dans un tube à centrifuger droit et soumis à centrifugation.

Les processus ci-avant décrits s'appliquent aux crèmes. Pour la séparation du gras de lait, nous procédons en ballons de 500 ou $1000 \mathrm{ml}$. Les proportions suivantes sont valables :

$200 \mathrm{ml}$ de lait,

$250 \mathrm{ml}$ de détergent,

$50 \mathrm{ml}$ d'eau à $90^{\circ} \mathrm{C}$.

Le procédé de séparation du gras par la mixture détergente donne généralement de bons résultats. La centrifugation est cependant nécessaire pour faciliter la séparation. On a constaté que le refroidissement préalable (une demi-heure au frigo, par exemple) des échantillons de produits frais facilite l'opération.

En effet, la matière grasse, non encore durcie, de laits (ou de crème) soumis à cette opération, peu d'heures après la traite, se sépare quelque fois plus difficilement.

\section{Divergences entre les résultats obtenus selon les divers modes opératoires.}

Quand on détermine le degré de lipolyse dans un même produit laitier en opérant selon les diverses méthodes décrites ci-dessus, on obtient des résultats quelque peu différents.

On constate toutefois un parallélisme assez rigoureux entre les résultats obtenus selon les méthodes $\mathrm{A}$ et $\mathrm{C}$. Par contre, les' teneurs en acides gras libres (FFA) déterminées selon la méthode B sont, d'une façon générale, beaucoup plus élevées et ce, surtout pour les échantillons de produits âgés et acides (acidité lactique).

Les divergences constatées s'expliquent de deux façons :

10 Par les conditions dans lesquelles s'effectue la titration de l'acidité présente dans l'extrait gras obtenu.

La matière grasse accompagnée des acides libres est dissoute dans un mélange de solvants et titrée par une liqueur alcaline, en présence d'un indicateur. 
Selon la quantité de gras mis en titration, la quantité et la nature des solvants utilisés, le titre et la nature de l'alcali employé, le point de virage admis, on obtient des valeurs assez diverses.

Il faut donc préciser, dans tous les cas, la méthode adoptée et ne comparer que des résultats obtenus en suivant un même mode opératoire. Nous préférons, pour notre part, déterminer l'acidité sur des prises d'essai assez importants ( 5 ou $10 \mathrm{~g}$ ).

$2^{\circ}$ Par les conditions dans lesquelles l'acidité libre est extraite du produit analysé en même temps que la matière grasse.

En effet, les acides gras qui accompagnent la matière grasse sont d'abord libérés et ensuite extraits différemment selon les processus suivis. Les acides gras libres sont solubles, en partie dans la matière grasse, en partie dans l'eau. D'autres acidités organiques, non liées à la matière grasse, peuvent également être extraites par les solvants utilisés (cas de la méthode B).

Ainsi, selon les techniques de séparation ou d'extraction utilisées, on détermine non seulement les acides gras solubles dans la matière grasse, mais éventuellement aussi, des proportions variables d'acides gras (ou autres) solubles dans l'eau.

Selon notre expérience les valeurs d'acidité, comparées à l'acidité de la matière grasse réelle du beurre obtenu, sont d'une façon générale :

1) A peu près parallèles, en adoptant la technique $A$,

2) Beaucoup plus élevées, en suivant le processus aux solvants B,

3) Quelque peu inférieures (environ 10 p. 100) en appliquant la méthode $\mathrm{C}$.

Pour situer ces divers niveaux, voici, à notre avis, les valeurs limites au-delà desquelles on peut considérer une matière grasse comme déjà nettement lipolysée :

Méthode $\mathrm{A}:$ : 0,9-1,2 $\mathrm{ml}$ alcali $\mathrm{N}$ par $100 \mathrm{~g}$ de matière grasse,

Méthode B : 2-3 $\mathrm{ml}$ alcali $\mathrm{N}$ par $100 \mathrm{~g}$ de matière grasse,

Méthode $\mathrm{B}$ : 0,7-1 $\mathrm{ml}$ alcali $\mathrm{N}$ par $100 \mathrm{~g}$ de crème normale,

Méthode C : 0,8-1,0 ml alcali $\mathrm{N}$ par $100 \mathrm{~g}$ de matière grasse.

La méthode classique au gel de silice (Harper) est fréquemment utilisée pour la détermination des acides gras libres dans les laits. On trouve plus rarement dans la littérature, mention de son application aux crèmes.

Cette technique qui donne des résultats concordants avec ceux obtenus selon la méthode B, aux solvants, nous paraît plus compliquée et plus dispendieuse (résultats selon méthode de Harper = $\pm 1,5 \times$ FFA (méthode B)).

Aussi nous n'y avons eu recours que comme méthode de référence : 
DEGRÉS DE LIPOLYSE DANS SIX ÉCHANTILLONS DE CRÈMES (exprimés en ml alcali $\mathrm{N}$ pour $100 \mathrm{~g}$ de matière grasse)

\begin{tabular}{|c|c|c|c|c|c|}
\hline Crèmes & \multicolumn{5}{|c|}{ Méthodes } \\
\hline Nos & $p \mathrm{H}$ & $\underset{(\mathrm{AMG})}{\mathbf{A}}$ & $\frac{\mathrm{B}}{(\mathrm{FFA})}$ & $\begin{array}{c}\mathrm{C} \\
(\mathrm{BDI} 2)\end{array}$ & $\begin{array}{c}\text { Gel de silice } \\
\text { (Harper) }\end{array}$ \\
\hline $1 \ldots$ & 6,70 & 0,72 & 1,05 & 0,64 & 1,66 \\
\hline 2. & 4,90 & 0,74 & 2,30 & 0,78 & 3,97 \\
\hline 3 & 5,15 & 0,76 & 2,60 & 0,69 & 4,83 \\
\hline $4 \ldots$ & 4,45 & 0,79 & 5,05 & 0,75 & 7,39 \\
\hline $5 \ldots$ & 4,35 & 0,92 & 5,05 & 0,88 & 7,08 \\
\hline $6 \quad \ldots$ & 4,40 & 0,88 & 4,00 & 0,83 & 6,19 \\
\hline
\end{tabular}

Le degré d'acidité de la matière grasse des beurres a été déterminé suivant une méthode analogue à celle normalisée par la FIL (toutefois nous employons la thymolphtaléine comme indicateur).

\section{II. - Degrés d'acidité de la matière grasse (AMG) dans les beurres}

Comme les traitements normaux, subis en beurrerie industrielle, par la matière première, ne devraient guère influencer les niveaux d'acidité de la matière grasse, le degré d'acidité d'un beurre devrait toujours refléter assez fidèlement le degré d'hydrolyse préexistant dans les crèmes ou les laits mis en œuvre. Or, les influences perturbatrices, si elles existent tendent toujours à accroître le degré d'acidité dans le produit fini.

Malgré ces restrictions, on a généralement considéré le degré d'hydrolyse de la matière grasse d'un beurre comme un test valable de frâ̂cheur de la matière première travaillée.

La plupart du temps, on constate en plus, une relation assez nette entre le degré d'acidité et la qualité du beurre. Il existe certainement des cas d'exception mais d'une façon générale, on peut admettre qu'un beurre à acidité de matière grasse basse est le plus souvent de bonne qualité parce que préparé de matière première toute fraîche. Par contre, un beurre à acidité de matière grasse moyennement élevée, peut être tantôt de qualité médiocre, tantôt de bonne qualité. Un beurre à haute acidité du gras sera 
affecté de certains défauts, parfois mineurs, mais le plus souvent nettement décelables. Dans bien des cas il sera franchement mauvais. Il est très rare, mais non exclu qu'un beurre à haute acidité puisse cependant encore paraître excellent.

Si l'on examine les diverses valeurs d'acidité de la matière grasse (AMG) des beurres belges, on constate deux faits :

1) Un relèvement du taux d'AMG au cours des mois d'hiver (phénomène explicable par un pourcentage plus élevé de vaches en état avancé de lactation, par l'alimentation hivernale et par une acidification moindre des crèmes fermières) ;

2) Un niveau moyen d'AMG plus élevé dans les beurres faits de crèmes fermières que dans les beurres préparés à partir de lait frais (lipolyse importante en cours de conservation à la ferme).

On peut admettre qu'un beurre présentant une AMG égale ou inférieure à 0,8 a été préparé à partir d'une matière première ne présentant aucune lipolyse notable. La plupart de ces beurres sont de plus, des beurres de bonne saison.

Lorsque les valeurs d'AMG atteignent ou dépassent 1,2-1,3, on estime que les beurres en question ont subi une lipolyse notable et le plus souvent préjudiciable à la qualité.

Toutefois, la dégradation de la qualité n'est pas toujours proportionnelle aux taux d'AMG.

\section{III. - Degrés de lipolyse dans les laits frais}

Comme nous l'avons longuement souligné au chapitre I, le degré de lipolyse observé dans les laits dépend de plusieurs facteurs

1) De la méthode d'appréciation utilisée ;

2) De la nature des laits :

laits de bonne saison ou de saison hivernale, laits de vaches normales ou anormales;

3) De la durée et des modalités de conservation des échantillons de lait avant analyse ; laits.

4) Des traitements mécaniques et thermiques subis par les

Aussi, convient-il, avant de donner un aperçu de quelques résultats d'analyses, de bien spécifier les méthodes de détermination utilisées.

Pour déterminer le degré de lipolyse dans les laits, nous avons adopté la méthode, décrite ci-avant, par emploi d'une mixture détergente (méthode C (BDI)). Deux modalités furent cependant utilisées successivement : 
1) Méthode $B D I[1]$ avec mixture détergente $\mathrm{n}^{\circ} 1$ (selon les prescriptions danoises) et titration de l'acidité de la matière grasse contenue dans une prise d'essai de $\pm 1 \mathrm{~g}$.

2) Méthode $B D I[2]$ avec mixture détergente telle que décrite ci-avant et titration de l'acidité de la matière grasse contenue dans une prise d'essai plus importante de l'ordre de 5 à $10 \mathrm{~g}$.

Les valeurs obtenues selon la méthode [1] sont plus élevées que celles constatées en adoptant le mode opératoire [2]. Rappelons que nos préférences vont cependant à la méthode BDI [2].

\section{Résultats de l'examen de divers laits obtenus et analysés sous diverses conditions.}

a) Laits normaux.

Laits frais ; analyse immédiate ; méthode [2].

(Traite du matin seule.)

Degrés de lipolyse (exprimés en ml alcali N/100 g de matière grasse) : 0,23 - 0,25 - 0,30-0,15 - 0,25 - 0,12 - 0,25.

Valeur moyenne (laits) : 0,23.

(Crèmes fraîches correspondantes : $0,43-0,44-0,49-0,50$ $0,51-0,48-0,44$.)

(Valeur moyenne (crèmes correspondantes à $30-35$ p. 100 de matière grasse) : 0,47 .)

b) Laits lipolysés (laits anormaux).

Laits frais; analyse immédiate ; méthode [2].

(Mélange des deux traites.)

Degrés de lipolyse : 0,55 - 0,85 - 0,38 - 0,56 - 0,58 - 0,60 - 0,40 $0,50-0,58-0,56$.

Valeur moyenne (laits) : 0,55.

(Valeur moyenne crèmes correspondantes : de l'ordre de 1,\%.)

c) Laits normaux; analyse différée ; méthode [1].

Deux traites; origines diverses ; échantillons conservés quelques heures au froid : $0,76-0,83-0,57-0,67-0,85-0,80-0,75-0,82$ 0,70 - 0,73.

Valeur moyenne : 0,75 (cf. rapport Hillerød, moyenne : 0,50-0,75).

d) Laits lipolysés; analyse différée ( $\pm 8 \mathrm{~h}$ au froid) méthode [1].

Une traite effectuée en tuyauterie avec agitation excessive : $1,00-0,95-1,46-1,43-1,03-1,32-1,38$.

Valeur moyenne : 1,22 (cf. rapport Hillerød : 0,75-1,39). 


\section{IV. - Degrés de lipolyse dans le lait, la crème correspondante, immédiatement après écrémage, puis après conservations de durées variables et sous diverses conditions}

Toutes les valeurs citées ci-dessous ont été obtenues selon la méthode C (BDI 2).

Selon cette technique, la lipolyse enregistrée dans des laits normaux (une traite) $\pm 0,23$ atteint habituellement le niveau de 0,47 dans de la crème fraîche à teneur normale en matière grasse (30-40 p. 100) et le niveau 0,41 dans de la crème claire (10-12 p. 100 de matière grasse).

En réalité, la matière grasse contenue dans de tels laits et crèmes est lipolysée de façon identique. Ce ne sont que les indices qui sont différents, d'où l'importance de toujours préciser les conditions d'analyses.

Par contre, la façon d'obtenir la crème d'un lait présente une grande importance. A titre d'exemple, citons les degrés de lipolyse enregistrés dans un lait, mélange des deux traites refroidies, et la crème obtenue après réchauffage de ce lait à bonne température d'écrémage (travail anormal à la ferme, normal en usine).

Lait normal (mélange des deux traites, dont celle du soir refroidie à $\left.\pm 2^{\circ}\right): \pm 0,45-0,55$.

Crème immédiatement après écrémage : 0,90-0,95.

La durée de conservation d'une crème crue, après écrémage ainsi que les conditions de températures subies (refroidissement ou réchauffage) jouent également un grand rôle.

Ainsi une crème fraîche (lait d'une traite) dont la lipolyse initiale se chiffre à 0,46 , voit celle-ci hausser jusqu'à 0,68 après conservation pendant 13 heures à 5-70 $\mathrm{C}$ (crème non acidifiée, $p \mathrm{H} 6,8)$.

Dans la pratique fermière, on conserve rarement les crèmes séparées.

Nous avons surtout examiné le développement de la lipolyse dans des crèmes accumulées pendant 7 jours (14 traites). Les crèmes étaient conservées de deux façons :

1) En cruches contenant, par portions de $20 \mathrm{~kg}$, les divers écrémages de la semaine (d'où la première cruche contient de la crème âgée de 6 à 7 jours et la dernière la crème la plus fraîche),

2) En vrac, toutes les crèmes étant mélangées dans une même cuve. 
Dans les deux cas, les lots de crèmes étaient refroidis et conservés aux températures respectives de $+1+2^{\circ} \mathrm{C}$ pour les cruches et de $+3+4^{\circ} \mathrm{C}$ pour le vrac.

Comme témoins, représentant le mode de conservation habituelle en cave, nous avons simultanément conservé des crèmes à $13^{\circ} \mathrm{C}$ (air) pendant 42 heures.

Les divers échantillons présentaient les degrés de lipolyse suivants :

Premier essai.

En cruches : la plus vieille $\ldots \ldots \ldots \ldots \ldots$ la plus récente :

\begin{tabular}{ccccc}
0,90 & 0,90 & 0,70 & 0,59 & 0,65 \\
\hline \multicolumn{5}{c}{ mélange : 0,68}
\end{tabular}

En vrac : mélange : 0,70 .

Témoin $\left(42 \mathrm{~h}\right.$ à $\left.13^{\circ} \mathrm{C}\right): 0,60$ (c'est-à-dire : $\left.0,76-0,55-0,42\right)$.

Deuxième essai.

En cruches : la plus vieille .......... la plus récente :

\begin{tabular}{rrrr}
0,78 & 0,70 & 0,57 & 0,47 \\
\hline mélange : & 0,72
\end{tabular}

En vrac : mélange : 0,65.

Témoin $\left(42 \mathrm{~h}\right.$ à $\left.13^{\circ} \mathrm{C}\right): 0,68$.

En conclusion de ces essais, on peut admettre que, à condition de refroidir les crèmes, une conservation de 7 jours n'entraîne pas une lipolyse exagérée. L'accumulation des crèmes au froid pendant une semaine donne d'aussi bons résultats que la conservation en cave pendant seulement 42 heures.

\section{V. - Degrés de lipolyse dans des crèmes fraîches et des crèmes âgées d'un à deux jours}

a) Valeurs constatées sur crèmes fraîches, une traite, analyse immédiate.

(Voir les tableaux à la page suivante).

Toutes ces crèmes, d'une traite, présentaient un $p \mathrm{H}$ normal $(6,7-$ $6,8)$ ainsi qu'une teneur en matière grasse moyenne (30-40 p. 100). 


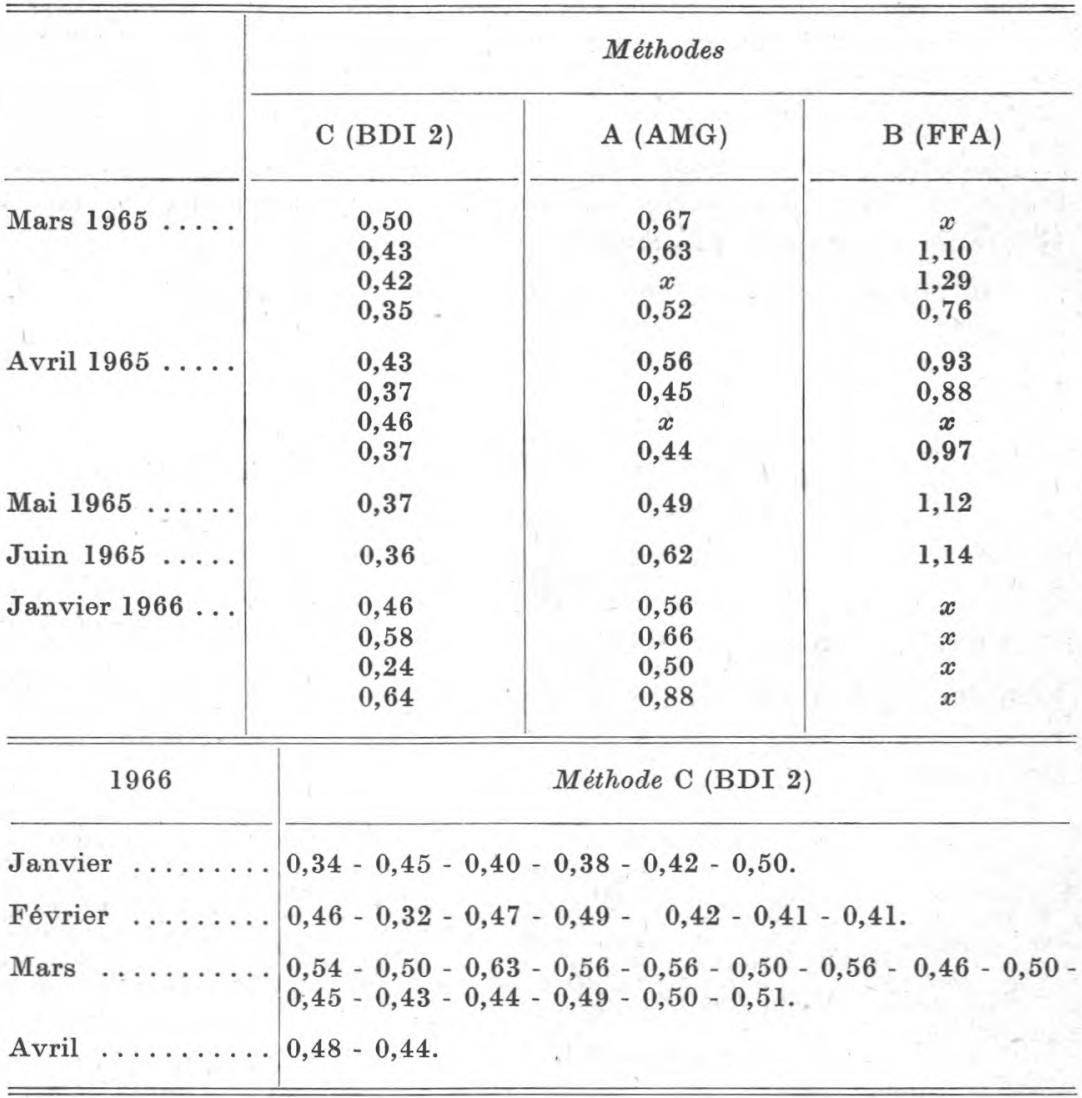

b) Valeurs constatées sur crèmes de un à deux jours (FFA).

On constate que la lipolyse s'accroît avec l'âge de la crème ainsi qu'avec l'acidification lactique.

\begin{tabular}{|c|c|c|}
\hline \multirow[b]{2}{*}{$p \mathrm{H}$ des crèmes } & \multicolumn{2}{|c|}{$\begin{array}{c}\text { Moyennes des } F F A \\
\text { (ml alcali } \mathrm{N} / 100 \mathrm{~g} \text { mat. gr.) }\end{array}$} \\
\hline & 32 heures & 56 heures \\
\hline$\geqslant 6,5$ & 1,12 & 1,47 \\
\hline $5,7-6,4$ & 1,56 & 1,72 \\
\hline $4,8-5,6$ & 1,69 & 1,82 \\
\hline$\leqslant 4,7$ & 1,74 & 1,92 \\
\hline
\end{tabular}




\section{VI. - Degrés de lipolyse dans des crèmes fermières collectées une ou deux fois par semaine}

(115 échantillons, mars à mai, valeurs moyennes et extrêmes) ( $\mathrm{ml}$ alcali $\mathrm{N}$ pour $100 \mathrm{~g}$ de matière grasse)

\begin{tabular}{|c|c|c|c|}
\hline$p \mathrm{H}$ & $\begin{array}{c}\text { Méthode } A \\
\text { AMG }\end{array}$ & $\begin{array}{c}\text { Méthode } B \\
\text { FFA }\end{array}$ & $\begin{array}{c}\text { Méthode } C \\
\text { BDI (1) }\end{array}$ \\
\hline $6,0-6,6$ & $\begin{array}{c}1,35 \\
0,76-3,68\end{array}$ & $\begin{array}{c}1,83 \\
1,10-4,42\end{array}$ & $\begin{array}{c}1,03 \\
0,53-2,90\end{array}$ \\
\hline $5,0-6,0$ & $\begin{array}{c}1,42 \\
0,74-4,38\end{array}$ & $\begin{array}{c}2,63 \\
1,20-5,69\end{array}$ & $\begin{array}{c}1,23 \\
0,54-3,35\end{array}$ \\
\hline $4,6-5,0$ & $\begin{array}{c}0,94 \\
0,61-1,67\end{array}$ & $\begin{array}{c}2,21 \\
1,42-3,78\end{array}$ & $\begin{array}{c}0,72 \\
0,37-1,02\end{array}$ \\
\hline $4,3-4,4$ & $\begin{array}{c}1,07 \\
0,44-1,67\end{array}$ & $\begin{array}{c}2,53 \\
1,47-3,58\end{array}$ & $\begin{array}{c}0,85 \\
0,30-1,32\end{array}$ \\
\hline
\end{tabular}

En examinant attentivement les tableaux précédents, on constate que, dans les vieilles crèmes :

1) Le $p H$ n'est pas toujours un indice valable de qualité de la matière grasse. De nombreux échantillons classés selon les $p H$ en première catégorie dénotent un degré de lipolyse particulièrement élevé (voir : colonne AMG (beurre)).

2) Le FFA semble nettement influencé par le $p \mathrm{H}$ des crèmes (aucune interférence semblable de l'acidification lactique (ou autre) sur les résultats des autres tests).

3) Cette caractéristique du FFA confère à ce test de lipolyse, la particularité de définir en même temps certaines conditions de conservation des crèmes puisque celles-ci modifient l'acidification du non-gras (surtout lorsque les résultats sont rapportés à $100 \mathrm{~g}$ de crème.)

4) Les tests de lipolyse ne révèlent cependant qu'un aspect de la qualité des crèmes. Toutefois, le degré d'altération de la matière grasse paraît primordial dans la fixation de la qualité d'une matière première destinée essentiellement à la fabrication du beurre (82 p. 100 de matière grasse).

\section{VII. - Appréciation, par les tests de lipolyse, de I'influence de diverses modalités de conservation des crèmes, à la ferme, sur la qualité de la matière première collectée en vue de la fabrication du beurre}

Une grosse part du beurre fabriqué en Belgique provient encore du travail industriel de crèmes fermières collectées deux à trois fois 
par semaine. L'autre partie de la production repose sur la récolte journalière de lait. Cependant la tendance actuelle est à la collecte de plus en plus générale de lait.

Comme, d'une part, le ramassage journalier de lait s'avère coûteux dans les régions à faible densité laitière et comme d'autre part, il importe de réduire les prix de revient du beurre, nous avons estimé utile d'étudier, du point de vue qualité, le travail, en beurrerie, de crèmes refroidies et conservées pendant une semaine.

Nous avons comparé la qualité des crèmes en fabrication, d'une part, crèmes accumulées en \pm 8 jours, en citerne réfrigérée $\left(+3^{\circ} \mathrm{a}+7^{\circ} \mathrm{C}\right)$ et d'autre part, crèmes refroidies de façon ordinaire (cave ou eau), conservées 3 à 4 jours. Ces essais s'étalent sur les mois de janvier à juin (période hivernale et estivale).

Les résultats enregistrés démontrent que :

1) Les degrés de lipolyse atteints, dans les crèmes conservées selon les modalités comparées, sont assez équivalents et rarement préjudiciables.

2) Le développement de l'hydrolyse du gras dans les crèmes conservées pendant au moins une semaine, au froid, n'est pas exagéré. Il paraît parfois plus intense, dans certains cas, sans qu'il soit toujours possible d'en déterminer les raisons réelles.

3) Les degrés de lipolyse semblent s'accroître brusquement au cours des premières 48 heures de conservation, se stabiliser ensuite pendant plusieurs jours, puis croître le plus souvent à partir des 6-7 ${ }^{\mathrm{e}}$ ou $8^{\mathrm{e}}$ jours de conservation. Cette dernière hausse du taux de lipolyse est souvent accompagnée d'un accroissement sensible de l'acidification $(p \mathrm{H})$.

En conclusion, on peut admettre que la conservation, au froid, de longue durée, n'entraîne pratiquement aucune lipolyse excessive, tout au moins lorsqu'on limite la période de conservation à 7 jours. A remarquer, cependant, que les crèmes ainsi traitées seraient, assez rarement, quelque peu plus sensibles à certains défauts d'oxydation (légère tendance à l'apparition du goût métallique dans le beurre sortant de baratte).

Du point de vue lipolyse, la conservation au froid de crèmes fermières pendant une semaine fournit, à l'industrie, une matière première de qualité comparable au système actuel de collecte deux ou trois fois par semaine. Les crèmes conservées au froid ne s'acidifient pas et ne nécessitent plus, par conséquent, de neutralisation avant pasteurisation. La conservation, en vrac, d'un mélange (lait plus crème) serait à étudier.

La confrontation des résultats obtenus au cours de ces essais avec ceux rapportés aux chapitres $V$ et VI permet de mieux apprécier tout l'intérêt de la conservation au froid, de longue durée. 
TABLEAU SYNTHETIQUE DES RESULTATS

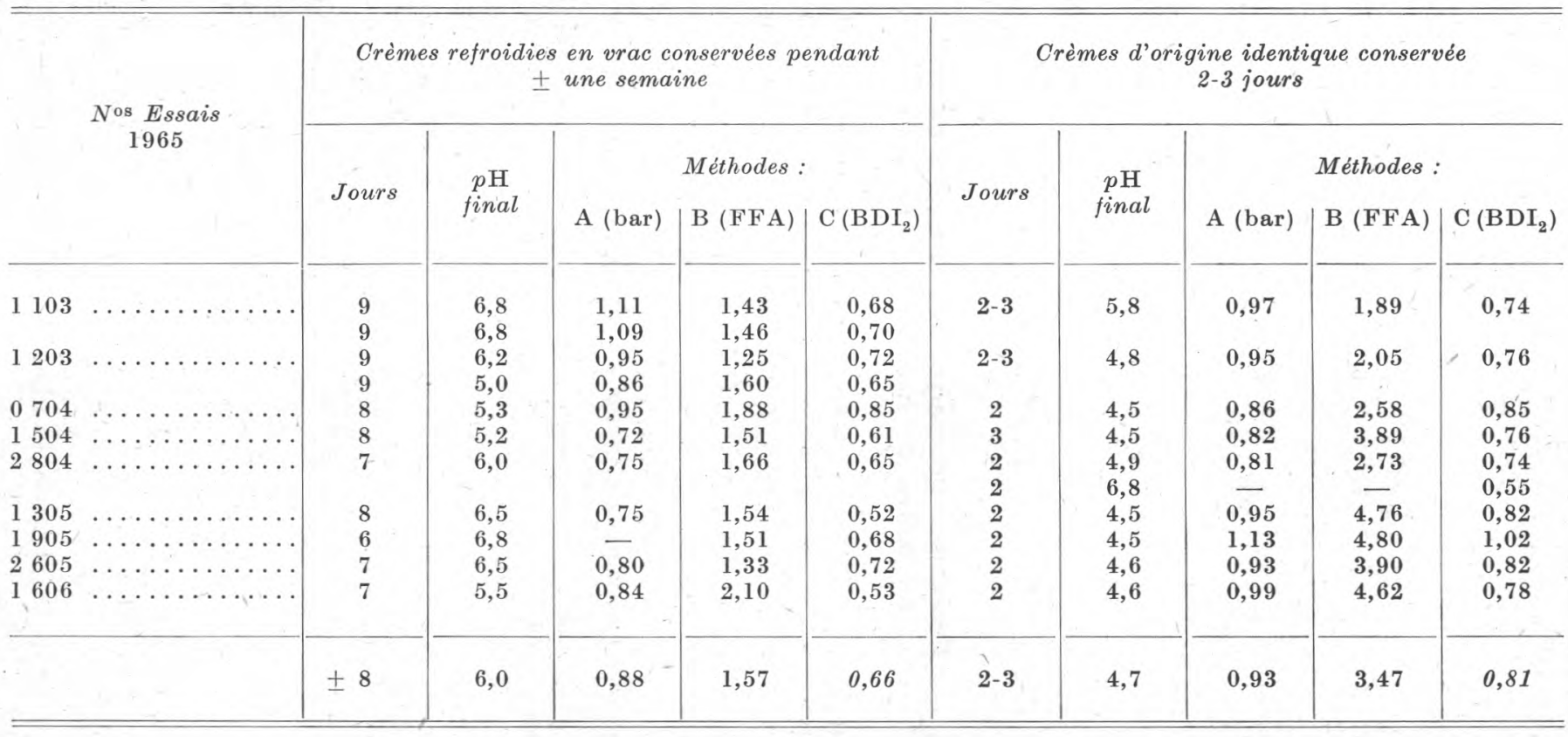




\begin{tabular}{|c|c|c|c|c|c|c|}
\hline 1966 & & & $\begin{array}{c}\text { Méthode } \\
\mathrm{C}(\mathrm{BDI} 2)\end{array}$ & & & $\begin{array}{c}\text { Méthode } \\
\mathrm{C}(\mathrm{BDI} 2)\end{array}$ \\
\hline 2001 & 9 & 5,8 & 1,08 & 4 & 6,8 & 0,86 \\
\hline 2801 & 8 & 6,3 & 0,94 & 4 & 6,7 & 1,04 \\
\hline 0402 & 7 & 6,7 & 0,54 & 4 & 5,7 & 0,76 \\
\hline 1602 & 11 & 4,9 & 0,95 & & & \\
\hline 0203 & 8 & 6,4 & 0,98 & & & \\
\hline 0903 & 7 & 6,4 & 0,72 & & & \\
\hline 2303 & 9 & 6,6 & 0,83 & & & \\
\hline \multirow[t]{2}{*}{0604} & 5 & 6,7 & 0,77 & & & \\
\hline & $\pm 8 \mathrm{j}$ & 6,2 & 0,85 & & & $\pm 0,89$ \\
\hline
\end{tabular}

(P. m. degré de lipolyse dans crèmes fraîches : 0,50 (une traite) à 0,75 (deux traites).)

\section{VIII. - Evolution du degré de lipolyse dans les crèmes en cours de fabrication du beurre}

Au cours de certains essais, nous avons apprécié l'influence des diverses opérations subies par les crèmes, sur l'hydrolyse du gras.

Brièvement, on peut résumer nos constatations comme suit :

1) La lipolyse est nettement plus élevée dans les mousses produites à la surface des crèmes conservées en cuves réfrigérées.

D'où la nécessité d'aménager les appareils de refroidissement et les manipulations de façon à réduire la production de mousses.

2) Les fluctuations importantes de températures subies par les crèmes crues sont toujours préjudiciables. Il faut éviter tout réchauffage ou refroidissement non justifié. De même, toute agitation excessive favorise le développement d'une lipolyse accrue.

3) La neutralisation de l'acidité lactique avant pasteurisation ne modifie en rien le degré de lipolyse des crèmes.

4) Le traitement thermique (pasteurisation) n'entraîne pratiquement pas de modification de l'hydrolyse de la matière grasse.

5) Par contre, dans certains cas, nous avons constaté, en cours de maturation des crèmes avec emploi de levain, une hausse parfois sensible, des taux de lipolyse. Dans d'autres cas, on ne constate aucune influence en cours de maturation.

6) Le barattage c'est-à-dire la transformation en beurre de la crème mâturée, ne change guère l'état de lipolyse. A remarquer toutefois que les méthodes de détermination sont différentes d'où obligation de se montrer prudent dans les appréciations. 


\section{Résumé}

Après quelques considérations sur l'action des lipases dans les laits et les crèmes, l'auteur décrit les méthodes qu'il a utilisées pour la détermination de la lipolyse dans ces matières premières de l'industrie beurrière.

Il rappelle l'importance de l'acidité de la matière grasse des beurres comme test de qualité et de fraîcheur.

Il signale les degrés de lipolyse constatés dans des laits frais et dans des crèmes correspondantes, selon trois méthodes : barattage, extraction aux solvants et séparation par mixture détergente.

Il donne également les teneurs en acides gras libres extraits, aux solvants, de nombreuses crèmes réceptionnées en beurreries.

Il relate ensuite des essais de conservation de crèmes fermières à basse température. Les degrés de lipolyse constatés dans des crèmes accumulées au froid, pendant une semaine, ainsi que dans les beurres produits, n'atteignent, en aucun cas, des valeurs préjudiciables à la qualité.

L'auteur en déduit que la collecte hebdomadaire de crèmes réfrigérées pourrait remplacer avantageusement le mode actuel de ramassage de crèmes non refroidies artificiellement, collectées deux à trois fois par semaine.

\section{Summary}

After a few considerations over the action of the lipases in milks and creams, the author describes the methods adopted for the determination of the lipolysis in raw products utilized in the butter industry.

He reminds the importance of the acidity of butterfat as test of butter-quality and -freshness.

He marks out the degrees of lipolysis observed in fresh milks and in the correspondent creams, according to three methods : churning, solvent-extraction and separation by detergent-mixture.

He gives alike the contents of free fatty acids, extracted from many farm-separated creams, received in the plant.

He relates afterwards some storage experiments of refrigerated farm-creams.

The degrees of lipolysis observed in the creams stored during a week so as in the butters, don't reach such values that the quality would be lowered.

The author concludes that the ordinary system of collecting the not cooled creams twice or three times a week should be replaced by weekly collecting of artificially cooled creams. 


\section{BIBLIOGRAPHIE}

[1] Stadhouders (Dr Ir J.). "Vetsplitsing in melk I et II ", Misset's Zuivel 70 (1964), no 51, p. 1260. - Misset's Zuivel 71 (1965), $\mathrm{n}^{\circ} 3$, p. 53.

[2] Alats (Ch.). Science du Lait, $2^{e}$ édit., SEP., Paris, pp. 64 et 406.

[3] Kuzdzal-Savoie (S.). Colloque de Rennes, juin-sept. 1962.

[4] Andersen (K. P.), KJaergaard Jensen (S. G.). "L'action lipasique dans le lait et quelques produits laitiers", 136 e Beretning, 1962, Hillerod, p. 10.

[5] Andersen (K. P., Hansen (K.). "Indice d'iode et de réfraction dans la matière grasse provenant de différents troupeaux ", $139^{\circ}$ Beretning, 1963, Hillerod, p. 6.

[6] LAMPERT (Lincoln M.). "Rapide separation of fat for pesticide residue analysis of milk products". Journal of Dairy Science, 47 (9), sept. 1964 , p. 1013.

[7] Frankel (E. N.), Tarassuk (N. P.). "An extraction-titration method for the determination of free fatty acids in milk and cream" Journ. of Dairy Science, 38, 1955, pp. 751-763.

[8] Harper (W. J.), Schwartz (D. P.), El Hagarawy (I. S.). "A rapid silica gel method for measuring total free fatty acids in milk ". Journ. of Dairy Science, 39, 1956, pp. 46-50.

[9] Bachmann (M.). "Beitrag zur Kenntnis der lipolytischer Fettapalting in Milch und Käse ". Promotionsarbeit, Juris., Verlag Zürich, 1960, p. 29.

[10] Sлostrom (G.). "Lipase problems in milk and dairy products". A review. Report no 58, Alnarp, 1959. 